\title{
Philosophiques
}

\section{La réponse de Régis à Huet concernant le doute cartésien}

\section{Thomas M. Lennon}

Volume 35, numéro 1, printemps 2008

Les valeurs de l'ironie (1) et le scepticisme à l'âge classique (2)

URI : https://id.erudit.org/iderudit/018248ar

DOI : https://doi.org/10.7202/018248ar

Aller au sommaire du numéro

Éditeur(s)

Société de philosophie du Québec

ISSN

0316-2923 (imprimé)

1492-1391 (numérique)

Découvrir la revue

Citer cet article

Lennon, T. M. (2008). La réponse de Régis à Huet concernant le doute cartésien. Philosophiques, 35(1), 241-260. https://doi.org/10.7202/018248ar

\section{Résumé de l'article}

La critique du cartésianisme formulée par Pierre-Daniel Huet à la fin du XVII siècle constitue l'un des événements les plus marquants de l'histoire du scepticisme à la période moderne. Cette critique se fonde sur l'arsenal des arguments sceptiques produits durant tout le XVII ${ }^{\mathrm{e}}$ siècle et pave la voie à la position anti-métaphysique des Lumières, qui commence avec Bayle et se poursuit avec les philosophes en passant par Hume. La réponse attendue des cartésiens à l'encontre de Huet est venue de Pierre-Sylvain Régis, auquel Huet va répondre à son tour à plusieurs reprises. Bien que la critique de Huet ait porté sur l'ensemble des thèmes spécifiques au cartésianisme (le doute méthodique, le cogito, la clarté et la distinction des perceptions, etc.), et bien que Régis ait répondu sur tous ces points, il n'en reste pas moins que le débat entre eux s'est essentiellement concentré sur le doute cartésien, que nous examinons minutieusement ici à la lumière de leur confrontation. 


\title{
La réponse de Régis à Huet concernant le doute cartésien"
}

\author{
THOMAS M. LENNON \\ University of Western Ontario \\ tlennon@uwo.ca
}

\begin{abstract}
RÉSUMÉ. - La critique du cartésianisme formulée par Pierre-Daniel Huet à la fin du XVII iècle constitue l'un des événements les plus marquants de l'histoire du scepticisme à la période moderne. Cette critique se fonde sur l'arsenal des arguments sceptiques produits durant tout le XVII siècle et pave la voie à la position anti-métaphysique des Lumières, qui commence avec Bayle et se poursuit avec les philosophes en passant par Hume. La réponse attendue des cartésiens à l'encontre de Huet est venue de Pierre-Sylvain Régis, auquel Huet va répondre à son tour à plusieurs reprises. Bien que la critique de Huet ait porté sur l'ensemble des thèmes spécifiques au cartésianisme (le doute méthodique, le cogito, la clarté et la distinction des perceptions, etc.), et bien que Régis ait répondu sur tous ces points, il n'en reste pas moins que le débat entre eux s'est essentiellement concentré sur le doute cartésien, que nous examinons minutieusement ici à la lumière de leur confrontation.
\end{abstract}

\begin{abstract}
The attack of Pierre-Daniel Huet on Cartesianism at the end of the seventeenth century was one of the most significant events in the history of skepticism in the early modern period. It capitalized on the building momentum generated by the use of skeptical arguments throughout the century, and it opened the way to the anti-metaphysical stance of the Enlightenment, beginning with Bayle and passing to the philosophes, including Hume. The inevitable Cartesian response to Huet came from Pierre-Sylvain Regis, to whom Huet then responded in various ways. Although Huet's attack had taken aim at all the themes with which Cartesianism has come to be associated (the method of doubt, the cogito, clarity and distinctness of perception, etc.), and although Regis replied on all these topics, the debate between them concentrated on Cartesian doubt, here closely examined in light of their debate.
\end{abstract}

L'attaque lancée contre le cartésianisme par Pierre-Daniel Huet à la fin du XVII ${ }^{e}$ siècle fut l'un des événements les plus significatifs de l'histoire du scepticisme du début de l'époque moderne. Elle tire sa force de l'élan généré par l'utilisation d'arguments sceptiques tout au long du siècle et ouvre la voie à la posture anti-métaphysique des Lumières initiée par Bayle puis transmise aux philosophes et à Hume qui, chose rare, a reconnu sa dette envers Huet. Le fait que cette attaque se soit pensée contre le cartésianisme, qui représentait à l'époque le système philosophique dominant, est en lui-même significatif. Mais cette attaque s'est de plus concentrée sur tous les thèmes auxquels le

\footnotetext{
* Traduction Jonathan Naud, revue par Sébastien Charles.
} 
cartésianisme a été peu à peu associés: le recours au doute, le cogito, la perception claire et distincte, etc.

Comme il l'a reconnu dans ses Mémoires, Huet a été lui-même attiré par le cartésianisme, mais un examen plus tardif et plus précis de cette philosophie l'a amené à y renoncer, la jugeant dénuée de fondement. La publication en 1674-1675 par Malebranche de La recherche de la vérité fut sans doute pour Huet l'occasion d'en reprendre l'examen minutieux, l'ouvrage tournant en ridicule les objets de sa quête intellectuelle qu'il chérissait le plus : l'histoire ancienne, l'étude des langues mortes, etc. Les indélicatesses de Malebranche à l'égard de la perspective antiquisante n'étaient rien d'autre au fond qu'une version extrême du discours cartésien assimilant l'histoire à un ramassis de préjugés dont l'emploi du doute devait nous débarrasser, chacun ayant à bâtir par lui-même l'édifice du savoir de novo. Dans la perspective de Huet, une telle attitude n'exprime rien d'autre que de l'orgueil, de l'arrogance et de la vanité1.

La Censura philosophiae cartesianae de Huet paraît pour la première fois en 1689. Suite aux encouragements promulgués par le sceptique Simon Foucher, Huet s'est mis à la rédaction de cet ouvrage avec l'intention de l'inclure dans un travail d'apologétique chrétienne plus large, qui devait aussi inclure les Alnetanae Questiones et le Traité philosophique de la faiblesse de l'esprit humain, ce dernier n'étant publié qu'à titre posthume ${ }^{2}$. Bien que le but explicite de l'ouvrage soit de montrer que raison et foi ne s'opposent pas, même si la foi doit bien sûr être considérée comme supérieure à la raison, les travaux de Huet de cette époque s'orientent vers le fidéisme, et se veulent en rupture avec l'œuvre de Descartes, qui conduit, selon Huet, à une opposition entre foi et raison, voire à une subordination de la foi à la raison. Et la critique porte plus largement contre le cartésianisme en général, comme l'ambiguité du titre de l'ouvrage le laisse penser, et plus spécifiquement contre Malebranche ${ }^{3}$.

La solidité de cette attaque portée contre Descartes explique sa large diffusion, tant en France qu'à l'étranger (l'ouvrage a été réédité cinq fois en cinq ans), et la nécessité, pour les cartésiens, d'y répondre. Huet souligne dans sa préface de la nouvelle édition de 1694 le caractère européen des réponses faites à son ouvrage, provenant tant de Eberhard Schweling, professeur à Brême, que de Johannes Schotanus, professeur à Franeker, ou bien d'Andreas Peterman, professeur à Leipzig, de Burchard De Volder, professeur à Leyde, et de nombreux autres encore. La réfutation point par point de la Censura, ou ce qui en tenait lieu de tentative, était même devenu un exercice scolaire pour les étudiants de l'Université de Leiden. Que ce soit par défaut ou bien par choix délibéré, ce fut Pierre-Sylvain Regis, celui que Huet surnommera plus tard le «Prince des cartésiens », qui releva le défi parmi les cartésiens français. Pasquier Quesnel a décrit son Système (1690) comme étant en partie une réponse

1. À ce propos, voir Lennon $(2003,1)$.

2. Cf. Lennon $(2005,1)$.

3. Pour plus de précisions à cet égard, voir Lennon (2003, 2). 
à Huet. Mais la principale contre-attaque de Regis a été plus justement sa Réponse à la censure de la philosophie cartésienne publiée en 1691. Il s'agit d'un document extrêmement important à la fois pour l'histoire du scepticisme et celle du cartésianisme, étant donné que l'intention de Regis n'est pas seulement de défendre Descartes, mais également les cartésiens.

En opposition avec certaines parties de la Censura, la Réponse de Regis se présente comme un texte ne contenant pas de provocations, réfutant en grande partie la censure de Huet paragraphe par paragraphe, tout en admettant qu'elle contient des objections qui sont "extrêmement fortes, et bien concertées ${ }^{4}$ ». Regis doutait en partie du succès littéraire de son entreprise: les réponses de cette sorte «sont difficiles [...] on s'ennuie d'ordinaire en les lisant, surtout quand la satire en est bannie, comme elle le sera toujours de tout ce que je ferai ${ }^{5} »$. Cette attitude est adoptée intentionnellement pour faire ressortir par contraste la différence avec celle de Huet. Ainsi, Regis prétend avoir renoncé en particulier à spéculer sur les motifs qui ont poussé Huet à s'opposer à Descartes, montrant qu'Huet lui-même avait échoué à le faire à propos de ce dernier, spécialement dans la mesure où il lui avait attribué de l'orgueil, de l'arrogance et de la vanité.

Comme cela était à prévoir, Huet prit à son tour la plume contre Regis. La réponse la plus importante qu'il ait proposée a été une réédition largement augmentée de la Censura en 1694, dans laquelle il critique point par point les réponses apportées par Regis. Pour être plus précis, disons plutôt qu'il a commencé à ébaucher une telle critique car, après l'avoir entreprise minutieusement, il l'interrompt subitement au troisième chapitre du livre II. À cette critique s'ajoute un manuscrit inédit de la taille d'un livre, intitulé Censure de la réponse faite par M. Regis au livre intitulé Censura philosophiae cartesianae, texte de 125 pages qui était apparemment considéré par Huet comme achevé et destiné à être publié tel quel. Il contient un index très détaillé, une page-titre stylisée avec pseudonyme, de même qu'une lettre de "l'éditeur ", de la main même de Huet, qui l'informe que, quand l'ouvrage lui a été envoyé de France, on ne lui a donné aucune raison expliquant son inachèvement. Et, effectivement, le manuscrit se termine également au chapitre trois du livre deux. Il n'est bien sûr pas anodin que les annotations de Huet sur sa propre copie de la Censura et sur sa copie de la Réponse de Régis s'interrompent au même point. Pourquoi cette interruption aussi abrupte qu'inattendue?

Comme on le verra par la suite, les enjeux philosophiques qui ont opposé Huet et Regis sont assez techniques et compliqués, et ils impliquent des incompréhensions de part et d'autre, mais également à l'égard de Descartes. Cela n'est sans doute pas étranger au fait que Huet se soit impatienté lors de sa lecture de Regis, retrouvant dans la Réponse ces traces d'orgueil, d'arrogance et de vanité qu'il avait déjà perçues chez Descartes et Malebranche. Il semble

4. Regis (1691), «Préface», p. 4.

5. Ibid. 
alors qu'il en soit venu à la conclusion qu'un changement de stratégie était nécessaire pour combattre plus efficacement le cartésianisme. Au lieu de discuter en termes techniques d'enjeux philosophiques difficiles, il s'est dit que recourir à la caricature et au ridicule valait mieux, suivant en cela l'exemple du Voyage dans la lune du jésuite Daniel.

Les Nouveaux mémoires pour servir à l'histoire du cartésianisme de Huet ont été écrits durant l'hiver 1691-1692, soit peu après la publication par Regis de sa Réponse. Ils sont dédiés à Regis, "Prince des cartésiens ", célèbre dans les rues et les salons pour être le protecteur de la matière subtile, le patron des globules et le défenseur des tourbillons. Le ton ironique de la dédicace sied bien à l'ouvrage. L'hypothèse soutenue par les Nouveaux mémoires est que, bien que l'on dise pourtant le contraire, Descartes est toujours vivant et qu'il enseigne la philosophie en Laponie où, par sa petite sature, sa barbe noire et son teint basané, il se fait passer pour un Lapon. Plaisanterie mise à part, Huet cite la biographie publiée récemment par Baillet où l'on peut lire que Descartes a quitté la Hollande pour la Suède en raison de la "pénible tâche d'être l'oracle du genre humain ${ }^{6}{ }^{\star}$. Ce qui est à noter ici en rapport avec les enjeux dont nous traiterons plus tard, c'est que Huet fait dire à Descartes que, bien qu'il sache que le cogito est un argument impliquant trois termes, il a préféré le présenter comme une proposition à deux termes afin de couper court à de possibles objections.

Ce changement de stratégie expliqué, reste à comprendre pourquoi Huet a malgré tout choisi de publier une seconde édition de la Censura en 1694. Dans la préface, il évoque le manque de respect dû à son travail et à sa personne pour justifier le fait qu'il ne pouvait laisser sans réponse la critique de Regis faite au nom des cartésiens. Il ajoute de plus qu'il a décidé de concentrer toute son attention sur le premier chapitre, car ce dernier rend compte du fondement du système cartésien, fondement dont le sapement suffit à entraîner la destruction de l'édifice en son entier. En bref, malgré le changement de stratégie de Huet, une réfutation philosophique du cartésianisme lui paraît une réponse adéquate à l'orgueil, l'arrogance et la vanité des partisans de ce système.

Huet ouvre le premier chapitre de la Censura de 1689 par l'affirmation suivante: «Le premier fondement de toute la philosophie de Descartes est qu'il faut douter, et douter non pas faiblement et légèrement, mais de manière que nous regardions comme incertaines et même comme fausses toutes les choses du monde ${ }^{7}$ ». La force effective et le sérieux de ce doute mènent à une objection immédiate quant à sa possibilité même. Selon Huet, douter de quelque chose consiste à suspendre son jugement à propos de sa valeur de vérité. En ce sens, il semble impossible de douter d'une chose et de la déclarer en même

6. Baillet (1691), p. 396; Huet (1692), p. 15.

7. Huet (1689), p. 11 (traduction manuscrite inédite de la Bibliothèque Nationale, verso de la p. 3). De l'édition de Paris de 1689 à l'édition de Paris largement augmentée de 1694, la Censura philosophiae cartesianae a connu quatre autres éditions quasiment toutes identiques, même au niveau de la pagination. 
temps fausse, et ce parce que si l'on considère que quelque chose est faux, cela implique que sa contradictoire est vraie.

La première chose qui mérite d'être censurée, c'est qu'afin de mieux douter il veut que l'on tienne toutes choses non seulement pour incertaines, mais pour entièrement fausses, ce qui est visiblement contraire à son dessein. Car un homme qui reconnait une chose pour fausse ne doute pas plus que celui qui la reconnaît pour vraie, puisqu'il juge qu'il croit et qu'il assure qu'elle est fausse, et que cependant, pour douter, il faut suspendre son jugement et balancer entre le faux et le vrai ${ }^{8}$.

Au final, Huet accepte malgré tout de considérer que Descartes ait pu vraiment douter et qu'il l'ait fait pour de très bonnes raisons, qu'il partage également. Cependant, puisque Descartes prétend rechercher la vérité, vérité qu'il est incapable de trouver au vu de la solidité des raisons motivant son doute, Huet pense qu'il est obligé de feindre qu'il doute parce qu'il sait qu'il ne pourra pas le surmonter. En résumé, Descartes est obligé de recourir à la dissimulation pour ne pas admettre l'inanité de son entreprise, et ce à cause de son " orgueil, de son arrogance et de sa vanité ». Il existe néanmoins de bonnes raisons de croire que la description de la démarche cartésienne proposée par Huet est largement inexacte?

Tout n'est pas faux dans le compte rendu de Huet: il est vrai que Descartes, pour des raisons qui seront discutées plus tard, considère certaines de ses croyances, et certaines seulement, comme fausses. Il s'agit des croyances évidentes, comme par exemple que deux et trois font cinq. Pour sa part, Regis nie toutefois que Descartes ait jamais mis en question cela. Il pense que l'évident n'est jamais considéré comme faux, mais qu'il est plutôt hypothétiquement mis en doute (avec tout ce qui est sujet au doute, ce qui inclut bien sûr les croyances fondées sur la perception sensible), afin d'en proposer un examen plus minutieux. Après cet examen, il est vraisemblable que certaines choses resteront indécidables ou seront peut-être rejetées comme fausses (comme il en va pour de nombreuses croyances fondées sur la perception sensible) et celles qui sont évidentes seront acceptées comme vraies. Nous nous attarderons plus tard sur cette réponse de Regis. Pour le moment, retenons que Regis semble conduit à une position de ce type parce qu'il accepte la caractérisation dichotomique du doute de Huet, qui fait de celui-ci soit quelque chose de réel, fondé sur de bonnes raisons, soit quelque chose de fictif, adopté en ce cas au nom de motifs indéfendables. Cela étant, le résultat en est que l'interprétation de Regis bute sur des évidences textuelles insurmontables, Descartes ne disant tout simplement pas ce que Regis lui fait dire, et sur des problèmes philosophiques dus à une nouvelle mécompréhension de Descartes.

8. Ibid., p. 13 (traduction BN, recto de la p. 4).

9. Cf. Lennon $(2005,2)$. 


\section{6 - Philosophiques / Printemps 2008}

On peut enfin se demander pourquoi Regis a accepté, comme cela semble être le cas, la caractérisation du doute formulée par Huet. À ce stade de l'analyse, remarquons que le doute peut être authentique, c'est-à-dire non feint, sans pour autant être réel, au sens propre et pratique du terme. Ou, plus précisément, le doute peut être mené au nom de raisons théoriques sans être pour autant destiné à tromper. Gassendi, chez qui Huet a peut-être puisé sa conception du doute, a dénoncé en Descartes un philosophe ayant recours au niveau de sa formulation du doute à "l'artifice, la prestidigitation et la périphrase ", car, si l'on peut douter de beaucoup de choses, «il n'y aura personne qui se persuade que vous soyez pleinement persuadé qu'il n'y a rien de vrai de tout ce que vous avez jamais connu, et que les sens, ou le sommeil, ou Dieu, ou un mauvais génie, vous ont continuellement imposé ${ }^{10}$ ». En outre, Gassendi devance, du moins de manière indirecte, la critique de Huet voulant que quelqu'un ne puisse douter de ce qu'il suppose être faux. En considérant toute chose comme fausse, Descartes n'est-il pas parvenu non tant à "se dépouiller d'un ancien préjugé [qu'à se] revêtir d'un autre tout nouveau ${ }^{11}$ » ? De toute façon, la réplique (typiquement déplacée) de Descartes à Gassendi illustre ce qu'il aurait probablement répondu à Huet :

Un philosophe ne serait pas plus étonné de cette supposition [de fausseté] que de voir quelquefois une personne qui, pour redresser un bâton qui est courbé, le recourbe de l'autre part ${ }^{12}$, car il n'ignore pas que souvent on prend ainsi des choses fausses pour véritables, afin d'éclaircir davantage la vérité, comme lorsque les astronomes imaginent au ciel un équateur, un zodiaque et d'autres cercles, ou que les géomètres ajoutent de nouvelles lignes à des figures données, et souvent aussi les philosophes en beaucoup de rencontres ${ }^{13}$.

L'analogie avec la géométrie, comme nous allons le voir un peu plus loin, va nous aider tout particulièrement.

Pour l'instant, notons que la réponse apportée par Descartes à la disjonction proposée par Huet entre, d'une part, assumer le doute ou la fausseté, et, d'autre part, refuser qu'ils puissent aller de concert, aurait été de refuser les deux branches de l'alternative. Car son but est de parvenir à mettre en place un moyen général qui puisse déboucher sur une certaine perception de la vérité. Dans le cas présent, la vérité que veut révéler Descartes est la plus fondamentale de toutes, à savoir que toutes nos perceptions claires et distinctes sont vraies. Pour ce faire, il commence par intenter le plus grand procès possible à cette

10. Gassendi, Cinquièmes objections, dans Descartes (1963), t. II, p. 708.

11. Ibid., p. 707.

12. Juste avant d'introduire l'hypothèse du malin génie dans la Première Méditation, Descartes affirme (étant donné l'enracinement de ses croyances) : «je pense que j'en userai plus prudemment, si, prenant un parti contraire, j'emploie tous mes soins à me tromper moi-même, feignant que toutes ces pensées sont fausses et imaginaires "; Descartes, Méditations, I, dans Descartes (1963), t. II, p. 411.

13. Descartes, Réponses aux cinquièmes objections, dans Descartes (1963), t. II, p. 790. 
hypothèse en affirmant que toutes nos perceptions claires et distinctes sont fausses. Nous devrions douter de toutes nos perceptions parce qu'elles sont fausses, sans pour autant nous engager à reconnaître la vérité de cette proposition.

Nous voyons ici une confirmation du fait que la doctrine des vérités créées constitue le cœur du système cartésien. Le 15 avril 1630, Descartes écrit à Mersenne qu'il a découvert les fondements de la physique en satisfaisant à l'obligation qui est faite à tout être pourvu de raison de chercher à connaître Dieu et à se connaître. Nous voyons ici une référence elliptique au procédé de la mise en doute radicale qui sera mise en œuvre par la suite dans le Discours et les Méditations. Cette conjecture est confirmée quand Descartes en vient à mentionner que son traité sur la physique devrait inclure "plusieurs questions métaphysiques, et particulièrement celle-ci : que les vérités mathématiques, lesquelles vous nommez éternelles, ont été établies de Dieu et en dépendent entièrement, aussi bien que tout le reste des créatures ${ }^{14}{ }^{4}$. Dans une lettre suivante, il explique que la causalité par laquelle Dieu crée les vérités éternelles est la même que celle qui lui permet de créer toutes les autres choses, «car il est certain qu'il est aussi bien auteur de l'essence comme de l'existence des créatures: or cette essence n'est autre chose que ces vérités éternelles ${ }^{15}{ }$. De plus, il ne semble y avoir aucune exception à la dépendance de toute vérité à l'égard de la volonté parfaitement omnipotente et indifférente de Dieu, ce qui vaut d'ailleurs aussi pour les vérités des mathématiques et de la logique (la loi de non-contradiction). Comme Descartes l'explique encore dans une lettre plus tardive à Mesland : "Dieu ne peut avoir été déterminé à faire qu'il fût vrai que les contradictoires ne peuvent être ensemble, et que, par conséquent, il a pu faire le contraire ${ }^{16}{ }^{\prime}$. Si la loi de non-contradiction peut être autre qu'elle est, alors, en cas de doute radical, une proposition peut être considérée comme fausse sans que sa contradictoire ne soit pour autant acceptée comme vraie.

Il faut savoir que cette doctrine de la création des vérités éternelles n'a pas été acceptée par tous les cartésiens. Fait le plus notable, elle a été rejetée par Malebranche qui pensait qu'un tel volontarisme radical conduirait à un scepticisme dont on ne pourrait sortir, puisqu'il serait impossible de jamais savoir ce que la liberté d'indifférence divine rendrait vrai à un moment donné du temps. Mais, ironie de l'histoire, la doctrine a été acceptée par Regis, de sorte que, s'il n'avait pas accepté la caractérisation du doute de Huet, il aurait pu soutenir, contre l'objection de ce dernier, que le doute et le fait de considérer comme fausse une croyance pour des raisons théoriques sont deux choses incompatibles. Autre ironie de l'histoire, Regis, sur un sujet relativement proche, a semblé comprendre la doctrine de l'induction cartésienne comme la manière idoine de pratiquer une intuition. L'enjeu en est le cogito,

14. Descartes, Lettre à Mersenne du 15 avril 1630, dans Descartes (1963), t. I, p. 259. 15. Descartes, Lettre à Mersenne du 27 mai 1630, dans Descartes (1963), t. I, p. 267. 16. Descartes, Lettre au Père Mesland du 2 mai 1644, dans Descartes (1963), t. III, p. 74. 
qui occupe en fait la plus grande partie du premier chapitre de la Censura, sur lequel nous souhaitons désormais nous pencher.

Selon Huet, "quoique Descartes ait insisté avec une affectation extraordinaire sur la nécessité de douter de toutes choses, il ne fait cependant point difficulté dans la suite d'affirmer avec l'air du monde le plus décisif mille choses obscures, incertaines, inconnues, dont il ne donne souvent que des raisons fort légères, et quelquefois il n'en donne point du tout ${ }^{17}{ }^{\prime}$. Ce que Huet considère chez Descartes comme un abandon gratuit du doute s'étend même jusqu'au cogito ${ }^{18}$, Descartes n'ayant jamais poussé le doute jusqu'à ses ultimes conséquences, se contentant d'éviter la question ou de s'enfermer dans un cercle vicieux.

La circularité de l'argument des Méditations, qu'Arnauld souligne particulièrement dans les Quatrièmes objections, est bien connue:

Il ne me reste qu'un scrupule, qui est de savoir comment il se peut défendre de ne pas commettre un cercle, lorsqu'il dit que 'nous ne sommes assurés que les choses que nous concevons clairement et distinctement sont vraies, qu'à cause que Dieu est ou existe'. Car nous ne pouvons être assurés que Dieu est, sinon parce que nous concevons cela très clairement et très distinctement; donc, auparavant que d'être assurés de l'existence de Dieu, nous devons être assurés que toutes les choses que nous concevons clairement et distinctement sont toutes vraies ${ }^{19}$.

En bref, à l'intérieur de cette longue argumentation de Descartes faite pour surmonter le doute sceptique et obtenir un savoir certain, l'existence de Dieu reste toujours une petitio principii. Huet reprend cette objection pour l'approfondir encore. Il pense que la première prémisse de Descartes, à savoir celle qui porte sur le cogito lui-même, est tout aussi problématique. Descartes n'est pas dans une position qui lui permette d'évoquer l'existence de Dieu ou la règle qui veut que ce que l'on perçoive clairement et distinctement comme vrai le soit en réalité, tout simplement parce qu'il ne peut même pas prouver qu'il existe lui-même.

Huet interprète le cogito comme contenant un participe modifiant un pronom dont on postule l'existence du référent : je suis (une chose qui est) pensant. De là découle le « je suis ", mais seulement si l'on prend pour acquis ce que Descartes prétend prouver. Or, Descartes n'existe que s'il pense, mais il ne pense que s'il existe. Ainsi il y a cercle dans le cercle ${ }^{20}$.

17. Huet (1689), pp. 2-3 (traduction $\mathrm{BN}$, recto de la p. 1).

18. Huet (1689), p. 31 (traduction BN, verso de la p. 9): « voici tout d'un coup cet homme irrésolu, qui veut qu'on doute de tout, changé dans un instant, et sans nous dire qui peut l'avoir assuré que Dieu ou quelque méchant génie ne l'abuse point, il affirme en homme pleinement convaincu qu'il pense, et qu'il est. Déterminé par cette seule raison qu'il est contradictoire que ce qui pense ne soit pas dans le temps qu'il pense, et après avoir une fois rompu cette barrière qu'il s'était faite lui-même, il établit comme des vérités constantes tout ce qu'il croit comprendre clairement, et tout ce qu'il s'imagine connaître par la lumière naturelle».

19. Arnauld, Quatrièmes objections, dans Descartes (1963), t. II, p. 652.

20. Huet (1689), p. 14. 
La réponse de Regis à ce paradoxe renvient à tracer une distinction rationnelle que Huet ne parvient pas à comprendre ou refuse d'admettre. Il se peut bien que le référent du pronom existe - ce qui est, après tout, le but de l'exercice - mais il doit cependant être conçu non comme existant, mais seulement comme pensant. Ainsi, la question ne se pose plus. En 1694, Huet se confronte au dilemme: si le référent est considéré comme une chose qui pense, alors il s'ensuit une tautologie vide selon laquelle une chose pensante est une chose pensante (comme lorsque Chrysippe affirmait qu'il fait jour, donc il fait jour). Mais si le référent est plutôt considéré comme une chose qui existe, on retrouve une petitio principii, et le fait qu'il pense n'est d'aucun intérêt ${ }^{21}$.

La distinction que Regis propose est du même genre que celle que Descartes nomme, dans ses Principes (I, 62), distinction de raison ou de pensée selon laquelle "nous distinguons quelquefois une substance de quelqu'un de ses attributs, sans lequel néanmoins il n'est pas possible que nous en ayons une connaissance distincte ${ }^{22}$ ». Par opposition avec la distinction réelle selon laquelle les choses distinctes (substances) peuvent être conçues comme existant indépendamment les unes des autres, et par opposition avec la distinction modale selon laquelle une chose (une substance) peut être conçue comme existant indépendamment d'une autre (un mode), et non l'inverse, une distinction rationnelle ne permet pas qu'un des deux termes puisse être conçu comme existant indépendamment de l'autre (une relation entre une substance comme le référent du pronom « je » et l'un de ses attributs, comme la pensée ou l'existence, ou une relation entre deux attributs de ce genre).

Dans une lettre essentielle à cet égard, Descartes insiste sur le fait que la distinction rationnelle, telle qu'il la comprend, a un fondement dans la réalité $^{23}$. Suivant une terminologie qu'il puise chez Suarez, il pense ici à une distinctio rationis rationatae et non à une distinctio rationis rationantis, ce qui revient à dire en fait que la distinction n'est pas seulement verbale, conventionnelle, mais qu'elle a un fondement dans la réalité. Ainsi, la pensée, l'existence et la substance à laquelle se réfère le pronom «je » sont réellement une même chose, mais la même chose conçue différemment. Et dire qu'elles sont conçues différemment ne revient pas à dire que les mots "pensée », " existence » et «Je » sont appliquées à la même chose. Descartes en reviendrait alors à la distinction rationis rationantis, ce que Descartes refuse. Le recours au cogito a pour fonction de faire comprendre que la pensée, l'existence et le référent du pronom «je » sont réellement une même chose. Le cogito ne pose donc pas problème et ne renvoie pas à un argument circulaire, parce que ce n'est absolument pas un argument. C'est une démarche destinée à actualiser une intuition.

21. Huet (1694), pp.16-17.

22. Descartes, Principes de la philosophie, I, 62, dans Descartes (1963), t. III, p. 131.

23. Descartes, «Lettre à $* * *$ »(1645 ou 1646), dans Descartes (1964), t. IV, pp. 348-350. 
Dans une perspective cartésienne, la distinction rationnelle fondée en réalité est employée fréquemment et avec beaucoup de succès en géométrie. Prenons la cinquième proposition, clairement intuitive, du premier livre des Éléments d'Euclide, à savoir que les angles à la base d'un triangle isocèle sont égaux. Pappus a contourné la preuve passablement compliquée d'Euclide en recourant à un apport supplémentaire qui lui a permis de parvenir à une preuve très simple :

Si l'on conçoit le triangle [isocèle] comme étant deux triangles [...] toutes les parties correspondantes (des triangles) sont égales, et même les deux côtés égaux et l'angle entre eux, et, dès lors [comme cela a été prouvé par la proposition 4] il en va de même pour les angles sous-tendus par les côtés égaux, qui sont les angles à la base du triangle ${ }^{24}$.

Mais pourquoi parler de deux triangles? Selon le commentaire classique de Thomas Heath :

Nous devons dire que nous ne conservons qu'un seul triangle, mais vu selon deux aspects. S'il s'agit d'aider un débutant à le comprendre, nous pourrions lui dire que l'un des triangles est celui qui est vu de face et que l'autre est le même triangle mais vu de derrière, mais tout ceci n'est même pas nécessaire ${ }^{25}$.

Pour le dire autrement, la construction d'un triangle isocèle consiste à construire un triangle dont les angles de la base sont égaux, et alors penser à l'un revient à penser à l'autre, bien que d'une manière différente, puisqu'ils sont la même chose. Ce qui est vrai concernant cette connaissance d'un triangle isocèle est vrai de toutes les autres démonstrations d'Euclide qui, du point de vue de Descartes, sont autant de démarches superflues dont la fonction est de nous amener à la perception d'un seul objet ${ }^{26}$.

À l'inverse, pour Huet, les distinctions rationnelles ne prouvent rien. Si la pensée et l'existence sont choses différentes, il existe une petitio; si elles ne le sont pas, aucune conséquence n'est bonne. La raison qui explique ce refus est sans doute le fait que, pour lui, la distinction de raison n'est autre, suivant la terminologie de Suarez, qu'une distinctio rationis rationantis, c'est-à-dire une distinction sans différenciation ou, plus simplement, une vérité linguistique. D'où l'identité de la prémisse et de la conclusion de l'argument de Chrysippe, qui sont conçues de manière semblable. Nous ne disposons tout simplement pas du genre de savoir réel que les cartésiens prétendent avoir concernant la substance et les attributs. Cependant, pour Regis, un tel savoir est bel et bien accessible : bien que les référents de la prémisse et de la conclusion du cogito soient identiques, ils sont conçus différemment de telle façon que nous soyons conduits de l'un à l'autre, tout comme l'aurait fait l'argument de Chrysippe

24. Heath (1956), vol. 1, p. 254.

25. Ibid.

26. Pour une application de ce modèle à la conception spinoziste de la substance et, plus généralement à celle des rationalistes, voir Lennon $(2005,3)$. 
s'il était passé de la brillance du soleil au fait qu'il fasse jour, même si ces deux idées sont identiques.

Dans les trois premières sections du livre I, Huet présente le doute comme le fondement de la philosophie de Descartes, évoque les raisons qui ont poussé Descartes à agir ainsi et tente de montrer comment il a été contraint d'abandonner immédiatement un tel fondement. On y trouve aussi des arguties apparemment triviales sur le fait de savoir si le fondement de l'édifice cartésien est le doute ou le cogito. Ce fondement, Regis le décrit ainsi : "par le fondement de la philosophie, Monsieur Descartes et tous les autres philosophes entendent une vérité simple et connue par elle-même sur laquelle toutes les autres vérités de la philosophie sont appuyées ${ }^{27}{ }^{2}$. Huet répond à cela en 1694 en affirmant qu'il entendait le terme dans le sens de Vitruve, comme " un lieu que l'on creuse de sorte qu'un fondement solide puisse y être édifiée ${ }^{28}$ ", à savoir une notion essentiellement négative. Qu'un principe puisse être négatif, c'est une position soutenue dans la Critique de la réponse par le recours à l'exemple du De officiis de Cicéron où le premier principe de la justice est de ne faire de mal à personne, le fait de faire du bien à autrui n'intervenant qu'en second ${ }^{29}$. L'argument de Huet revient à dire que la fonction du doute est de supprimer les préjugés afin de rebâtir l'édifice du savoir.

Les enjeux sont importants dans ce désaccord apparemment superficiel. La question est de savoir si le doute est supposé révéler, chez le douteur, un soubassement déjà existant de certitudes dans l'ordre du savoir ou s'il a plutôt pour fonction de rendre sa construction possible. Si on lit Descartes correctement, comme Regis le fait, alors c'est la première hypothèse qui est la bonne. $\mathrm{Si}$ on le lit en s'inspirant de la tradition empirico-sceptique, ce que fait Huet, il faut lui attribuer l'autre branche de l'alternative. Regis perçoit avec finesse que Descartes souscrit peut-être au point de vue platonicien selon lequel à chaque étape du processus gnoséologique nous connaissons déjà tout ce que nous connaîtrons par la suite. Dans la Cinquième Méditation, après avoir examiné l'idée distincte qu'il a de l'étendue des corps, Descartes poursuit ainsi :

la vérité se fait paraître avec tant d'évidence et s'accorde si bien avec ma nature, que lorsque je commence à les découvrir, il ne semble pas que j'apprenne rien de nouveau, mais plutôt que je me ressouviens de ce que je savais déjà auparavant, c'est-à-dire que j'aperçois des choses qui étaient déjà dans mon esprit, quoique je n'eusse pas encore tourné ma pensée vers elles ${ }^{30}$.

27. Regis (1691), p. 2. Quelques pages plus loin, Regis affirme la même chose, à savoir que c'est le cogito et non le doute qui est le fondement de la philosophie de Descartes, et il ajoute que Huet lui-même en convient puisqu'on peut lire dans la Censura : " [Descartes] établit donc pour fondement de toute sa philosophie, cette proposition : 'Je pense; donc je suis' "; Regis (1691), pp. 9-10; Huet (1689), p. 13.

28. Huet (1694), p. 9.

29. Censure de la défense de la philosophie cartésienne faite par M. Regis, pp. 5-6 du manuscrit.

30. Descartes, Méditations, V, dans Descartes (1963), t. II, p. 470. 
Pour Huet, à l'inverse, une telle connaissance est acquise ou par héritage (rôle de la tradition) ou par construction (rôle de l'expérience). C'est l'antithèse exacte de la position de Descartes sur deux points précis. D'abord, la méthode cartésienne du doute élimine la tradition au profit de l'autorité de chaque individu. Quand il est accusé par les amis de Clerselier, et notamment Gassendi, de faire de sa propre pensée l'étalon de la vérité des choses, Descartes répond: " au sens que ces mots doivent ici être entendus, je dis que la pensée d'un chacun, c'est-à-dire la perception ou connaissance qu'il a d'une chose, doit être pour lui la règle de la vérité de cette chose ${ }^{31} »$. Ensuite, pour Descartes, toute construction est ipso facto une erreur. Gassendi pensait que l'idée de Dieu n'était pas innée, contre ce qu'affirmait Descartes, mais construite à partir d'éléments divers dérivés de l'expérience sensible. La réponse de Descartes est cinglante:

l'idée représente l'essence de la chose, à laquelle si on ajoute ou diminue quoi que ce soit, elle devient aussitôt l'idée d'une autre chose: ainsi s'est-on figuré autrefois l'idée d'une Pandore; ainsi ont été faites toutes les idées des faux dieux par ceux qui ne concevaient pas comme il faut celle du vrai Dieu ${ }^{32}$.

Et, chez Descartes, ce qui est vrai de l'idée de Dieu l'est, mutatis mutandis, pour toutes les autres idées.

Il est difficile de savoir quelle proportion de cette dispute qui apparaît insignifiante fut réellement comprise, à supposer qu'ils l'aient souhaité, par les protagonistes en question. Mais une chose est sûre, leur confrontation à l'égard du doute est loin d'être insignifiante. Comme l'écrit Huet:

il faut douter, et douter non pas faiblement et légèrement, mais de manière que nous regardions comme incertaines et même comme fausses toutes les choses du monde [...] il veut que nous doutions généralement de tout, sans laisser à notre esprit rien de certain sur quoi il puisse s'appuyer ${ }^{33}$.

Selon Regis, Huet se trompe en soutenant que Descartes considère toute chose comme incertaine et même comme fausse. La démarche de Descartes concerne plutôt une catégorie restreinte de choses qui sont véritablement incertaines. En réalité, Descartes a montré que seules les choses les plus évidentes (sans doute celles que nous percevons clairement et distinctement comme vraies) devraient être mises en doute, et uniquement afin de déterminer leur statut de vérité. Il y a donc deux sortes de doute. L'un d'eux est, pour reprendre les différentes qualifications que lui donne Regis, un doute feint, méthodologique, hypothétique, hyperbolique ou métaphysique, qui devrait être appliqué à tout. Grâce à lui, il est possible de faire un examen approfondi de toute chose et de se demander ainsi si ce dont on doute est vrai ou faux. Dans ce cas, on détermine peut-être moins la vérité des choses examinées sous les conditions de l'incertitude qu'on ne certifie ce qu'on sait déjà, avec pour finalité de manifester

31. Lettre de M. Descartes à M. Clerselier, dans Descartes (1963), t. II, p. 844.

32. Descartes, Réponses aux cinquièmes objections, dans Descartes (1963), t. II, p. 816.

33. Huet (1689), p. 11 (traduction BN, verso de la p. 3). 
un doute similaire au cas où on serait de nouveau confronté à un savoir incertain. C'est une manière de s'assurer de ce que l'on sait. Cela étant, ce doute dépend entièrement de la résolution de chacun "d'examiner de nouveau, non seulement tout ce qu'il a cru savoir, mais encore tout ce que se présentera à l'avenir à son esprit pour emporter son jugement ${ }^{34}$.

Le second type de doute «se tire de la nature même des choses qui ne se manifeste pas assez à notre esprit pour y produire des idées claires et distinctes $^{35}$ ». Les choses doivent donc être regardées comme incertaines, voire fausses. Il est plutôt vraisemblable que ce second type de doute soit, ou devrait être, un état permanent.

En ce qui concerne cette restriction à l'égard de ce qui doit être considéré comme faux, Regis renvoie, sans citer le texte, aux Principes (I, 2) qui, dans la version latine, se lit comme suit:

Il sera même fort utile que nous rejetions comme fausses toutes celles où nous pourrons imaginer le moindre doute, afin que si nous en découvrons quelquesunes qui, nonobstant cette précaution, nous semblent manifestement vraies, nous fassions état qu'elles sont aussi très certaines, et les plus aisées qu'il est possible de connaître ${ }^{36}$.

Le caractère plausible de la restriction de Regis dépend de l'antécédent de "ces choses » évoquées dans le précédent article, qui n'est guère plus long. Dans cet article, Descartes commence par remarquer que la longue habitude que nous avons eu de nous fier aux préjugés propres à la connaissance sensible nous éloigne du véritable savoir, puis rappelle que : «il n'y a point d'apparence que nous puissions nous en délivrer, si nous n'entreprenons de douter une fois en notre vie de toutes les choses où nous trouverons le moindre soupçon d'incertitude $^{37}$ ». La question est alors de savoir si ce moindre soupçon de doute peut être écarté par les opinions fondées sur les données sensibles, et la réponse est clairement non. Dans le troisième article, Descartes distingue ce doute, qui concerne seulement la contemplation de la vérité, de celui qui se manifeste dans la vie quotidienne, puis, dans les deux articles suivants, il l'applique à la fois à ce qui est perçu par les sens et aux démonstrations mathématiques. Le texte auquel Regis se réfère, quand on le replace dans le contexte des premières sections des Principes, ne permet pas de valider la restriction qu'il opère, mais plutôt de la refuser. Cela étant, il existe bien une réelle distinction entre le traitement que propose Descartes des données sensibles et celui des mathématiques, distinction sur laquelle nous reviendrons plus tard.

En ce qui concerne le doute qui, portant sur ce qui est clairement et distinctement perçu, se présente comme feint, méthodologique, hypothétique, hyperbolique ou métaphysique, et dépendant de notre volonté de douter, Regis

34. Regis (1691), p. 4.

35. Ibid., p. 3.

36. Descartes, Principes de la philosophie, I, 2, dans Descartes (1963), t. III, pp. 91-92.

37. Ibid., p. 91. 
évoque les Principes (I, 30), mais de nouveau sans citer le texte. Il s'agit d'un passage plus long intitulé: «Et que par conséquent tout cela est vrai que nous connaissons clairement être vrai, ce qui nous délivre des doutes ci-dessus proposés ». Les mots introductifs du titre indiquent bien que la référence proposée par Regis ne répond en rien à Huet. Les dix-sept articles précédents expliquent comment il se fait que "[la pensée] ne saurait avoir aucune science certaine, jusqu'à ce qu'elle ait connu celui qui l'a créée ${ }^{38}{ }^{\prime}$. Ce qui veut dire que c'est par la démonstration de l'existence d'un Dieu vérace que le doute est supprimé, ce qui permet de reconnaître comme vrai ce qui est clairement perçu. Mais avant cette démonstration, comme le texte l'indique clairement, les vérités mathématiques ainsi que toute vérité qui procède de l'expérience sensorielle peuvent être semblablement mises en doute. Mais une distinction est néanmoins faite entre la manière de traiter les sens et les mathématiques, qui se trouvait exprimée plus clairement encore dans les Méditations.

Pour le moment, on peut se demander comment Regis a pu être si borné sur un sujet aussi important dès le début de sa réponse à Huet. Mais il n'est pas le seul en cause, sa lecture ayant été adoptée par deux des plus notables commentateurs de Descartes au XX $\mathrm{XX}^{\mathrm{e}}$ siècle. Gilson voit deux sortes de doute chez Descartes : il doute réellement de ce qui semble vraiment douteux, puis il étend volontairement ce scepticisme à ce dont il ne doute vraiment pas (dans ce dernier cas, il imagine seulement des raisons qui lui permettent de concevoir la possibilité abstraite de douter $\left.{ }^{39}\right)$. Gueroult, citant Regis, ne fait que le paraphraser: «le doute méthodique et systématique, qui est feint et procède, non des choses, mais de la résolution de douter, diffère du doute véritable qui résulte des choses et peut engendrer le scepticisme ${ }^{40}$ ».

Qu'il l'ait pensé sérieusement ou non, Regis affirme que les attaques à l'endroit de Descartes, du style de celle de Huet, «plus digne[s] de pitié que de réponse », n'ont apporté aucun nouvel argument qui n'ait été déjà réfuté par Descartes lui-même ${ }^{41}$. La récente hypothèse voulant que Regis ait emprunté la précédente restriction de la réplique de Descartes aux Septièmes Objections de Bourdin peut être mise en lien avec ce qui vient d'être dit $^{42}$. Regis ne cite pas ici la réponse de Descartes à Bourdin, il ne le fera d'ailleurs pas plus dans le reste de la Réponse, mais comme cette réponse réitère son insistance à faire que le doute métaphysique et hyperbolique ne soit pas utilisé " pour les choses qui regardent la conduite de la vie ${ }^{43}$ ", cette interprétation est à première vue plausible. Mais une lecture serrée du texte ne permet pas de soutenir la restriction présentée par Regis. Elle renvoie bien à une distinction, mais qui est contraire à celle proposée par Regis.

38. Ibid., I, 13, p. 99.

39. Gilson (1925), p. 286.

40. Gueroult (1953), t. I, pp. 40-44.

41. Cf. Régis (1691), «Préface», p. 3.

42. Schmaltz (2002), p. 229.

43. Descartes, Septièmes réponses, dans Descartes (1963), t. II, p. 958. 
Descartes évoque deux points principaux ${ }^{44}$. Le premier renvoie au fait que Bourdin donne l'impression de croire que les « raisons solides (validas rationes) » de douter s'appliquent aux questions de la vie pratique, comme celle de savoir s'il a un corps, ce qui peut conduire les lecteurs imprudents à s'interroger sur son état mental, alors que des raisons de ce genre concernent en premier lieu des sujets qui sont difficiles à remettre en question. Il semble que le point de vue de Descartes soit de dire que la puissance de la raison qui doute est inversement proportionnelle à la difficulté de douter. Les préjugés dus aux sens peuvent être facilement mis en doute et ne nécessitent que de faibles raisons; les opinions mathématiques sont plus difficiles à remettre en cause et requièrent des raisons plus conséquentes. Nous reviendrons par la suite sur ce point. Notons en attendant qu'il n'existe pas de différences dans les manières de douter, mais plutôt dans le genre de raisons qui conduit au doute.

Le second point renvoie au fait que Bourdin se trompe quand il affirme que rien n'est épargné par le doute, signifiant par là que le doute, s'il est légitime, ne peut jamais être surmonté. Descartes y répond en disant que le doute de la Première Méditation est légitime et sensé (legitimae ac validae), parce qu'à ce moment précis il ne sait pas encore que ce que l'on perçoit clairement est vrai. Comme nous le savons, une fois l'existence de Dieu démontrée, cet obstacle est éliminé et le doute est surmonté. Encore une fois, on ne trouve pas de suggestion laissant penser que ce doute soit autre que véridique.

Ce que Descartes feint, c'est la fausseté de ce qu'il sait pouvoir être remis en doute. Et, dans ce cas, contrairement à sa réplique concernant le doute, Regis semble marquer un point en présentant comme hypothétique la fausseté que Descartes attribue aux choses incertaines. Au début de la Seconde Méditation, Descartes dit qu'il va prendre ses distances à l'égard « de tout ce en quoi je pourrai imaginer le moindre doute, tout de même que si je connaissais que cela fut absolument faux ${ }^{45}{ }$. C'est le chemin qu'il emprunte à la fin de la Première Méditation lorsqu'il invoque le malin génie, sa finalité étant alors la suivante: "j'emploie tous mes soins à me tromper moi-même, feignant que toutes ces pensées sont fausses et imaginaires ${ }^{46}{ }$. En reprenant consciemment ce qui est indubitablement la démarche des pyrrhoniens, celle que caractérise l'image de la balance équilibrée, Descartes tente ici de contrebalancer le poids des opinions préconçues et des mauvaises habitudes conduisant à des jugements erronés avec ce qui tient lieu de perception correcte. Le traitement que réserve Descartes à ses croyances antérieures (par exemple que deux et trois font cinq est une proposition fausse) n'est pas hypothétique, dans le sens où cela implique que la croyance est fausse afin de voir où peut aller un argument fondé sur elle en tant qu'elle est fausse - comme cela se fait de manière plus catégorique dans le cas d'une reductio ad absurdum, afin de démontrer

44. Ibid., t. II, pp. 957-961.

45. Descartes, Méditations, II, dans Descartes (1963), t. II, p. 414.

46. Ibid., t. II, p. 411. 
logiquement que la croyance est vraie. En fait, comme Gouhier l'a montré de manière persuasive, l'hypothèse du malin génie est plutôt d'ordre psychologique, comme le langage de Descartes le laisse penser ${ }^{47}$. Cette hypothèse prise en compte, afin de parvenir à une vérité quelconque, Descartes ne doit pas donner à ces anciennes opinions "plus de créance, qu'[il] ferait à des choses qui [lui] paraîtraient évidemment fausses ${ }^{48}$, ce qui le conduit à reconnaître aussitôt que, puisque ces croyances sont encore là, le résultat du doute est insuffisant. Il doit "prendre soin de s'en souvenir " ${ }^{49}$, et c'est pourquoi il invoque l'hypothèse du malin génie. Cet obstacle psychologique est la raison expliquant pourquoi, comme le dit le texte latin, il prend « un parti contraire » et « se trompe » lui-même ${ }^{50}$.

Dès lors, la règle méthodologique de Descartes est la suivante : trouver des raisons de douter de toute croyance avec pour limite le moment où la croyance paraît certaine (en faire moins laisserait intacte la certitude non fondée de la croyance, en faire plus générerait la croyance contraire qui n'est pas plus fondée - les deux plateaux de la balance doivent s'équilibrer). Et on doit chercher ces raisons jusqu'à ce qu'aucune raison ne donne lieu au doute concernant une croyance qui, dans ce cas, devrait être acceptée comme vraie, telle qu'elle doit l'être en fait. À proprement parler, le fait de considérer une croyance comme tout à fait fausse, par opposition à ce qui est plus ou moins douteux, est un cas limite de cette règle qui apparaît dans la Quatrième Méditation. Descartes rend compte ici du jugement qui produit la croyance. Sa thèse cherche à montrer que la volonté est contrainte par l'évidence, avec pour résultat que la liberté et la spontanéité de la croyance sont inversement proportionnelles à l'indifférence de la volonté. Il oppose la connaissance de sa propre existence découverte par la pensée, et le doute concernant la nature corporelle ou non de la substance pensante. L'évidence disponible à ce stade de l'argumentation ne favorise aucune des deux alternatives et le laisse donc dans un état d'indifférence. Et même si l'évidence de points de vue concurrents n'est pas égale, l'indifférence peut être atteinte. Le simple fait que cette conjecture est fondée sur une évidence incomplète peut être suffisant pour entraîner l'assentiment contre elle. Et Descartes de conclure: "Quod satis his diebus sum expertus, cum illa omnia quae prius ut vera quammmaxime credideram, propter hoc unum quod de iis aliquo modo posse dubitari deprehendissem, plane falsa esse supposui ${ }^{51} »$. Ce n'est pas un texte facile à interpréter (la traduction anglaise courante le fait d'ailleurs mal), mais le Duc de Luynes a bien su rendre le propos de Descartes: "J'ai posé pour faux tout ce que j'avais tenu auparavant pour très véritable, pour cela seul que j'ai remarqué que l'on en pouvait douter en

47. Gouhier (1962), p. 121.

48. Descartes, Méditations, I, dans Descartes (1963), t. II, pp. 410-411.

49. Ibid., p. 411.

50. Ibid.

51. Descartes, Meditationes de prima philosophia, IV, dans Descartes (1963), t. II, p. 211. 
quelque sorte ${ }^{52}$ ». Toutes ses croyances antérieures ne provoquent pas une telle réaction extrême le conduisant à les considérer comme fausses, mais seulement celles qui, vraisemblablement, ont la plus grande crédibilité, comme la croyance que deux et trois font cinq.

La même lecture doit être faite du début de la Seconde Méditation où Descartes, préoccupé par la profondeur des doutes qu'il a soulevés, se résout, comme nous venons de le voir, à prendre ses distances à l'égard « de tout ce en quoi je pourrai imaginer le moindre doute, tout de même que si je connaissais que cela fût absolument faux ${ }^{53}{ }$. Le chemin sur lequel s'achemine de nouveau Descartes n'est guère la large avenue des doutes commençant avec les sens, ouverte au doute maximal étant donné les arguments de tous ceux qui l'empruntent, parce que traiter les croyances fondées sur eux comme totalement fausses troublerait l'équilibre de la balance nécessaire à la suspension du jugement. Descartes s'engage plutôt dans la direction la plus neuve prise par le doute métaphysique ou hyperbolique minimal (reposant sur de fortes raisons) de croyances du type deux et trois font cinq. C'est dans cette sorte de doute que Descartes est tombé et il se sait en eaux troubles puisqu'il ne peut "ni assurer [ses] pieds dans le fond, ni nager pour [se] soutenir au-dessus ${ }^{54}$ ». Le doute portant sur les sens est d'un genre totalement différent; au contraire du doute métaphysique, il n'est jamais surmonté, sauf en termes de cohérence, et, pour des raisons pratiques, il ne peut être maintenu. C'est la raison pour laquelle Descartes affirme que son choix de se duper lui-même en manipulant sa volonté grâce à l'hypothèse du malin génie ne peut aller bien loin et n'est guère dangereuse, "puisqu'il n'est pas maintenant question d'agir, mais seulement de méditer et de connaitrere ${ }^{55}$.

Comme le faisaient les anciens sceptiques avec l'epôché, son accusation de fausseté ne va pas jusqu'à considérer comme vraie la contradiction de ces points de vue. Comme il l'explique à Bourdin :

Lorsque j'ai dit qu'il fallait pour quelque temps tenir les choses douteuses pour fausses, ou bien les rejeter comme telles, j'ai donné si clairement à connaître que j'entendais seulement que, pour faire une exacte recherche des vérités tout à fait certaines, il ne fallait faire non plus de compte des choses douteuses que de celles qui étaient absolument fausses ${ }^{56}$.

Le but est de neutraliser ces points de vue tout comme leur contradictoires.

Pour sa part, Huet n'a guère été impressionné par le recours à la fausseté hypothétique qu'invoque Regis ${ }^{57}$. Dans l'édition de 1694, il soutient que douter hypothétiquement d'une chose n'est pas moins incompatible avec le fait

52. Méditations, IV, dans Descartes (1963), t. II, p. 464.

53. Méditations, II, dans Descartes (1963), t. II, p. 414.

54. Ibid.

55. Méditations, I, dans Descartes (1963), t. II, p. 411.

56. Descartes, Septièmes Réponses, dans Descartes (1963), t. II, p. 959.

57. Huet (1694), p. 14. 
de la tenir pour hypothétiquement fausse que douter réellement de cette chose ne l'est avec le fait de la tenir réellement pour fausse. On peut alors prétendre que des propositions contradictoires sont contradictoires dans tous les mondes possibles, ce qui revient à dire qu'il n'existe aucun monde possible dans lequel elles seraient vraies toutes les deux. Ainsi, considérer une croyance comme fausse, même seulement d'un point de vue hypothétique, c'est considérer sa contradictoire comme vraie, et même si le point de vue est là aussi hypothétique.

Il est possible que les motivations de Huet tiennent à sa perception d'une malhonnêteté qu'il juge caractéristique des cartésiens. Un honnête homme doute seulement de ce qui est incertain, et ne considère comme faux que ce qui l'est ou, à tout le moins, qui lui semble tel. L'hypothèse de la fausseté, tout comme le doute hypothétique, sont une tricherie. Bien entendu, Descartes n'est pas un tricheur. Son doute est bien réel, même s'il ne se propose qu'un objectif théorique en y recourant. Il ne le feint pas; il ne trompe personne, et lui-même encore moins en dépit du langage qu'il utilise. Le seul souci de la vérité le pousse à prendre « un parti contraire » et à employer «tous [ses] soins à [se] tromper $[\text { lui-même }]^{58} »$.

En quoi cela change-t-il quelque chose que les croyances fondées sur les données sensibles soient considérées comme fausses et non seulement comme douteuses? Il est un cas, bien sûr, où elles ne peuvent pas être simplement considérées comme fausses, parce qu'elles sont en fait fausses, et ce de manière nécessaire. La douleur que je ressens au pied n'est pas dans mon pied. C'est seulement un mode de mon esprit. Mais à moins que je sente cette douleur comme présente dans mon pied, je ne me comporterai pas de manière à conserver l'unité du corps et de l'esprit. Maintenant, si la raison qui m'amène à douter si la douleur dans mon pied est aussi forte que la raison qui me conduit à douter que deux et trois font cinq, je n'arriverai peut-être jamais à l'attitude appropriée à cet égard. Invoquer l'hypothèse du Dieu trompeur en rapport avec le statut de la douleur pourrait ne pas fonctionner alors qu'il en irait différemment pour les mathématiques. Ainsi, Descartes s'oppose au portrait que trace de lui Bourdin en le présentant comme quelqu'un qui applique des « raisons solides » aux questions de la vie pratique, ce qui est en effet injuste.

En règle générale, nous pouvons récuser les croyances sensibles inadéquates en recourant à d'autres croyances du même genre, comme lorsque nous en venons à la conclusion que le bâton partiellement plongé dans l'eau qui apparaît courbé est en réalité droit. En dernier ressort, une telle correction consiste simplement à maintenir la cohérence des croyances. La croyance voulant que, en dépit des apparences visuelles, le bâton soit vraiment droit convient avec ce que les autres et nous voyons et avec ce que nous savons être vrai concernant le comportement d'objets de ce genre. Rappeler l'hypothèse d'un Dieu trompeur ruinerait cet effort vers la cohérence. En résumé, la valeur persistante de la perception sensible serait menacée si on la supposait totalement

58. Méditations, I, dans Descartes (1963), t. II, p. 411. 
fausse au lieu de la considérer comme simplement douteuse sur la base de fondements pratiques faillibles qui sont juste suffisants pour des objectifs théoriques.

En somme, en pensant que le savoir peut être atteint, Descartes pose bien une distinction entre deux sortes de doute, qui sont tous deux réels : un doute pratique qui influence le comportement et un doute théorique qui a pour but de déboucher sur l'évaluation de certaines vérités métaphysiques. En tant que sceptique, Huet interprète cette distinction comme fonctionnant entre doutes réel et feint, et la lie à des questions morales (orgueil, arrogance et vanité). Regis accepte cette distinction, et il est du coup amené à se méprendre sur la méthode dubitative de Descartes. Comment ce champion du cartésianisme, qui pense comme Descartes que le savoir peut être atteint, peut-il tant se méprendre?

On peut spéculer sur le fait que Regis n'ait fait que tirer les conséquences de la doctrine des vérités créées qu'il a puisé chez Descartes. Si toute vérité relève de la volonté indifférente de Dieu, il ne peut y avoir de garantie a priori pour quoi que ce soit, et nous devons au bout du compte nous en remettre à l'expérience et non à la raison. Bien que cette interprétation puisse ne pas être unanimement partagée ${ }^{59}$, les inclinations empiristes de la philosophie de Regis sont évidentes et hors de toute controverse. Ce que nous avons vu laisse à penser qu'il semble considérer le doute comme un procédé empirique, alors que, chez Descartes, il relève de l'ordre des raisons, d'une question de logique, ayant pour but de confirmer les intuitions fondées. De toute façon, le résultat en est que cette défense de la méthode dubitative cartésienne a dévié de manière telle qu'elle a pavé la voie menant au scepticisme empirique du XVIII ${ }^{\mathrm{e}}$ siècle.

\section{Bibliographie:}

Baillet, A. La vie de M. Descartes, Paris, D. Horthemels, 1691.

Descartes, R. Euvres philosophiques, Paris, Garnier, 1963-1973, 3 vol.

- CEuvres, Paris, Vrin, 1964-1974, 11 vol.

Gilson, E. Le Discours de la méthode de Descartes : texte et commentaire, Paris, Vrin, 1925.

Gouhier, H. La pensée métaphysique de Descartes, Paris, Vrin, 1962.

Gueroult, M. Descartes selon l'ordre des raisons, Paris, Aubier, 1953, 2 tomes.

Heath, T., dir., The Thirteen Books of Euclid's Elements, New York, Dover, 1956, 3 vol. Huet, P.-D. Censura philosophiae cartesianae, Paris, D. Horthemels, 1689 (réédition augmentée Paris, Jean Anisson, 1694).

—. Nouveaux mémoires pour servir à l'histoire du cartésianisme, Paris, s.n., 1692.

—. Censure de la philosophie de Descartes, Bibliothèque Nationale de France, manuscrit fonds français 14702.

- Censure de la réponse faite par M. Régis au livre intitulé Censura philosophiae cartesianae, Bibliothèque Nationale de France, manuscrit fonds français 14703. 
Lennon, T. M. «Malebranche, Huet and the Birth of Skepticism », dans G. Paganini, dir., The Return of Skepticism : From Hobbes and Descartes to Hume, Dordrecht, Kluwer, 2003, pp. 149-165 [2003, 1].

-. "Foucher, Huet and the Downfall of Cartesianism », dans T. M. Lennon., dir., Cartesian Views: Essays presented to Richard A. Watson, Leiden, E.J. Brill, 2003, pp. 117-128 [2003, 2].

—. "The Skepticism of Huet's Traité de la foiblesse de l'esprit humain ", dans M. A. Bernier et S. Charles, dir., Scepticisme et modernité, Saint-Étienne, Publications de l'Université de Saint-Étienne, 2005, pp. 65-75 [2005,1].

—. "Huet on the Reality of Cartesian Doubt ", dans T. Schmaltz, dir., The Reception of Descartes's Philosophy, London, Routledge, 2005, pp. 63-79 [2005, 2].

- "The Rationalist Conception of Substance » dans A. Nelson, dir., A Companion to Rationalism, Oxford, Blackwell, 2005, pp. 12-30 [2005, 3].

Regis, P.-S. Réponse au Livre qui a pour titre P. Danielis Huetii Episcopi Suessonensis designati, Censura Philosophiae Cartesianae, servant d'éclaircissement à toutes les parties de la Philosophie, surtout à la Métaphysique, Paris, Jean Cusson, 1691. Schmaltz, T. Radical Cartesianism, Cambridge, Cambridge University Press, 2002. 